\title{
Experimental Investigation of the Boundary Layer Transition on a Laminar Airfoil Using Infrared Thermography
}

\author{
Tomáš Jelínek ${ }^{1, *}$ \\ ${ }^{1}$ VZLU - Czech Aerospace Research Centre, Beranových 130, 19905 Prague, Czechia
}

\begin{abstract}
The transition in the boundary layer is investigated using infrared thermography (IRT). The study is carried out on a laminar airfoil in the transonic intermittent in-draft wind tunnel. The transition in the boundary layer is evocated using transition-generator strips of different thicknesses at two Mach numbers: 0.4 and 0.8 . The tested transition-generators thickness to boundary layer displacement thickness ratio was from 0.42 to 1.25. The Reynolds number respect to the airfoil chord is: $R e=1.0-1.4 \cdot 10^{6}$. The six cases for different transition-generators thickness ratios were compared. The behaviours of laminar and turbulent boundary layers are discussed. The use of IRT has been proven to be an appropriate tool for detecting the transition of the boundary layer in high-speed wind tunnel testing.
\end{abstract}

\section{Introduction}

To study the development of the boundary layer and the transition from the laminar to the turbulent flow in the boundary layer is very important because it fundamentally changes the character of the boundary layer; it means the parameters of the boundary layer and its behaviour. How the boundary layer behaves especially in critical flow regimes depends mainly on its nature and how the boundary layer is developed.This is important both in aerospace applications where the airplane's lift depends on the separation of the boundary layer from the wing airfoil and in the applications in the drive units and other turbomachinery applications of various power aggregates where the performance and efficiency of these machines depends on the nature of the boundary layer and its possible separation.

For these reasons, it is necessary to address the boundary layer's nature, to examine under what conditions and how soon the transition of the laminar boundary layer into turbulence occurs.

This phenomenon can be monitored, for example, by measuring the surface tension by means of hot wire anemometry. It requires equipping the surface of the object under investigation with hot films and to measure their response to cooling caused by fluctuations of the turbulence flow.

However, the formation of the transition of the boundary layer into turbulence also depends on the surface of the model under investigation, where small inequalities or changes on the surface may cause or bypass the transition of the boundary layer into turbulence. Thus, if the model is of small dimensions, it is problematic to equip the surface of such a model with sensors that always have the final dimensions, and so the nature of the boundary layer can be significantly affected. In these cases, it is appropriate to use a method that would detect the transition in the boundary layer without having to change the surface of the examined model.

One of the methods commonly used to visualize the transition of the boundary layer without any need to change the model's surface is visualization using high resolution infrared thermography. Its use is widespread in both aerodynamic wind tunnel testing and realconditions experiments.

In aerodynamic wind tunnel testing, it is sometimes necessary to artificially trigger the acceleration of the turbulence in those cases where the measurement results are significantly affected by the existing laminar boundary layer. Very often, the strips with roughness elements are used at varying distances at the leading edge of testing airfoil or wings when there is a need to fix the boundary layer transition.

This can also be the case of prismatic blades testing when inlet flow conditions (low inlet turbulence intensity along with a low Reynolds number) can cause the boundary layer to remain laminar up to the blade's trailing edge. This may not be the case with the real operation conditions of the blades, where the transition may take place much closer to the leading edge due to different inlet flow conditions.

The transition in the boundary layer can also be simulated using various mathematical models in Computational Fluid Dynamics (CFD). Today mathematical solvers are available that use different transition models and predict the transition in the boundary layer based on the boundary conditions. The

\footnotetext{
Corresponding author: jelinek@vzlu.cz
} 
obtained characteristics of boundary layers experimentally are therefore important also for validation of these mathematical models [1], [2].

To gain a better idea of how to use the transitiongenerator strips and how they can affect the transition in the boundary layer, an experiment was performed on the laminar airfoil in the transonic testing section at two Mach numbers and three different strips thickness.

\section{Principle of IR thermography}

There are many options how to detect the transition in the boundary layer experimentally. These methods are clearly described in [3]. For qualitative transition evaluation, the hot-wire anemometry (hot-films) is best to use. This method gives a very good conception of the behaviour of the boundary layer, but the installation of the surface sensors may, in particular in the case of small dimensions of the model, significantly affects the transition in the boundary layer.

Therefore, if there is need to determine the transition in the boundary layer, for example, for comparing (validating) with a CFD calculation, which uses transition models in the boundary layer, using IR thermography appears to be very good method.

The principle of detecting the character of boundary layer using high resolution IR thermography is a common technique in many research areas that has been used since the 1960s.

Scanning the infrared radiation one can detect both the transition to turbulence and the separation of the boundary layer. Increased heat transfer in turbulent flow compared to laminar leads to a change in the surface temperature of the model. It works providing that the fluid and the surface of the model have different temperatures. This technique is applicable to stationary measurements where the conditions of the boundary layer are stable in the long term, but it is also possible to measure unsteady phenomena with sufficient equipment.

The basic principle of IR thermography for detecting the transition of laminar boundary layer into turbulence is the difference in coefficient of convection of heat transfer between the laminar and turbulent boundary layers.

The laminar boundary layer acts as an insulator in the heat transfer between the ambient air and the surface of the model. On the other hand, in the turbulent boundary layer the vorticity of the flow increase and thus the heat transfer between the surrounding flow and the surface of the model increase as well. This will cause that the model surface temperature change. The surface temperature gets closer to the temperature of the flow.

Thanks to this phenomenon, the transition region can be detected as an area of steep temperature change between two areas with different temperatures. (The direction of temperature change is depending on whether the model is warmer than the ambient air or vice versa). The greater the difference between the temperature of the model and the flow, the more readable is the area of the transition.
IR thermography of turbulence boundary layer detection is therefore a temperature measuring technique related to forced thermal convection on the surface of the model under investigation. Heat transfer $q$ can be calculated using the heat transfer coefficient $h$, the stagnation temperature of the flow $T_{0}$ and the model surface temperature $T_{w}$ :

$$
q=h \cdot\left(T_{w}-T_{0}\right),
$$

where $h$ is closely related to the friction coefficient $c_{f}$, the velocity of the flow $v$, thermal conductivity of fluid $\lambda_{c}$ and kinematic viscosity $v$. For the heat transfer coefficient in the distance $x$ the dependence is given by:

$$
h(x)=1 / 2 \cdot c_{f}(x) \cdot v(x) \cdot \lambda_{c} / v .
$$

Equation (1) is often called the Newton's cooling law, equation (2) is determined from the Reynolds's analogy, which refers to the heat (energy) transfer in the boundary layer when the value of Prandtl number is close to one.

Usually, $c_{f}$ is one order higher for a turbulent case than a laminar one. If the heat transfer from the model to the flow $q$ (in the case of a warmer model's surface and a cooler flow) is kept constant (eg. by constant electrical heating), then if the $c_{f}$ increases (transition from laminar flow to turbulent), the surface temperature of the model $T_{w}$ will decrease. The resulting change in surface temperature of the model can then be measured, for example, by IR camera.

\section{Configuration of experiment}

\subsection{Wind tunnel}

The experiments were carried out in the transonic wind tunnel with testing section dimensions $0.4 \mathrm{~m}$ (width) and $0.8 \mathrm{~m}$ (height). It is an intermittent in-draft wind tunnel, which employs a vacuum chamber with a capacity of about $4100 \mathrm{~m}^{3}$, in which a vacuum is produced using a set of water-ring vacuum pumps. The maximum level of vacuum is about $40 \mathrm{kPa}$ of absolute pressure. Once the necessary pressure has been reached, the vacuum chamber is connected to atmospheric pressure and atmospheric air flows through the test section and through the adjustable throat. The humidity of intake air can be reduced in the wind tunnel inlet by a dryer plant. The air in the test section had a temperature of $284-287$ ${ }^{\circ} \mathrm{K}\left(11-14{ }^{\circ} \mathrm{C}\right)$; the surface temperature of the model was between $15-18{ }^{\circ} \mathrm{C}$ during the performed experiments.

The advantage of using an intermittent aerodynamic tunnel in this case is that the model does not need to be permanently heated to a different temperature because it still keeps the ambient temperature of the aerodynamic tunnel test section area. Exposure the model to the flow of lower temperature (since it was measured in winter time with a lower air temperature without switching on the air dryer) was only about 5 seconds. There was no change for the model to keep the ambient flow temperature during the time of wind tunnel run. 


\subsection{Model}

For the aerodynamic wind tunnel tests, the airfoil NACA 16-012 was selected. Geometry is visible in figure 1 . The model chord was $100 \mathrm{~mm}$; the span of the model was $200 \mathrm{~mm}$.

This is a symmetric laminar airfoil with a maximum thickness in $60 \%$ of the chord. The static pressure distribution on the surface of the model was available from the previous measurement.

\subsection{Transition-generator strips}

To accelerate the boundary layer transition on the laminar airfoil, the transition-generator strips were attached to the observed side of the model at a distance $x / c=0.35$ from the leading edge. Strips were made using the scotch tape of different thicknesses. The width of the strips was always $2 \mathrm{~mm}$. The three different thicknesses of the transition-generators, $K=0.04,0.08,0.11 \mathrm{~mm}$ were tested in total.

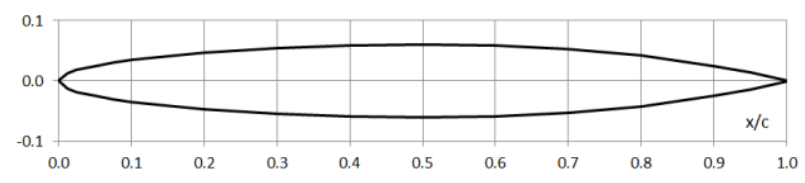

Fig. 1. Geometry of tested airfoil NACA 16-012.

\subsection{China clay visualization}

In addition to monitoring the boundary layer transition by means of IR camera, the surface visualization method was applied in the experiments using an oil mixture and visualization particles. Air kerosene (kinematic viscosity at $20^{\circ} \mathrm{C}$ is $1.3 \mathrm{~mm}^{2} / \mathrm{s}$ ) was used as oil.

Magnesium oxide powder was used as the visualization particles.

The mixing ratio was in all cases the same in a volume ratio of 1:4 (one unit of particle to four units of oil).

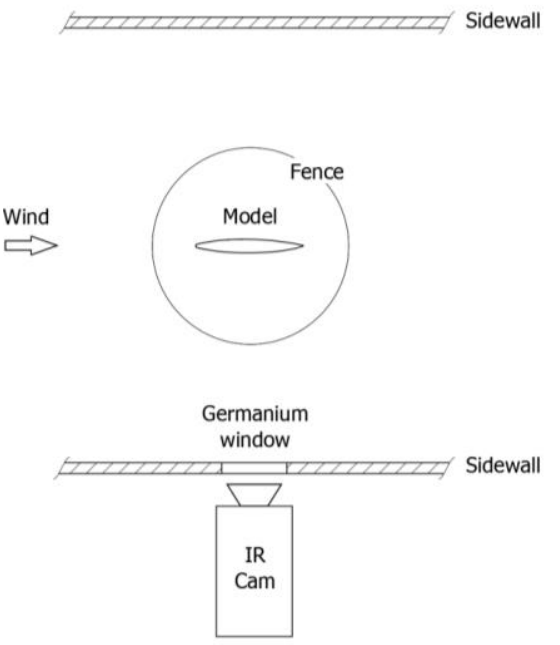

Fig. 2. Schema of the xperiment layout in the wind tunnel test section ( $0.8 \mathrm{~m}$ height, $0.4 \mathrm{~m}$ width)

\subsection{IR Thermocamera}

The high-resolution FLIR A655sc thermocamera was used for the experiment. The resolution of the video taken is $640 \times 480$ pixels collected at $50 \mathrm{~Hz}$. The camera's thermal resolution is less than $50 \mathrm{mK}$. The temperature measurement accuracy is $\pm 2{ }^{\circ} \mathrm{C}$.

For the data post-processing the ResearchIR ${ }^{\odot}$ software was used.

\subsection{Experiment layout}

Since it was necessary to provide optical access to the surface of the model, the model was placed in the test section in a non-standard manner, parallel to the side walls of the wind tunnel (see figure 2). It was necessary to attach the model to special brackets that were attached to the upper and lower walls of the test section. Since the model was not bounded by walls in the tunnel, the airfoil was equipped with circular fences of radius $r=170 \mathrm{~mm}$ made of $1 \mathrm{~mm}$ thick metal sheet.

From the sidewall of the wind tunnel, it was possible to capture the surface temperature of the model with a thermocamera through a germanium-glass window.

\section{Numerical simulations}

To imagine the thickness of the boundary layer, numerical 2D flow calculations were performed for two inlet Mach numbers (0.48 and 0.81$)$. By calculation, the boundary layer parameters were found in $35 \%$ of the profile chord, where the transition-generators were placed on the surface of the model.

The calculation was done using CFD code Edge [4].

The numerical simulation was performed as a laminar flow with fixed position of transition to turbulence at $60 \%$ of the chord length.

For the numerical simulation, the inlet conditions parameters were set as follows: $p_{0}=100,000 \mathrm{~Pa}, T_{0}=$ $288.15 \mathrm{~K}, \alpha=0^{\circ}$

The calculation results are summarized in table 1.

Table 1. Boundary layer parameters in $x / c=0.35$

\begin{tabular}{|c|c|c|}
\hline & $\boldsymbol{M}_{\infty}=\mathbf{0 . 4 8}$ & $\boldsymbol{M}_{\infty}=\mathbf{0 . 8 1}$ \\
\hline$\delta[\mathrm{mm}]$ & 0.340140 & 0.277734 \\
\hline$\delta^{*}[\mathrm{~mm}]$ & 0.094918 & 0.087988 \\
\hline$\delta^{* * *}[\mathrm{~mm}]$ & 0.044597 & 0.038229 \\
\hline$H[1]$ & 2.128346 & 2.301966 \\
\hline$\tau_{w}\left[\mathrm{~kg} \cdot \mathrm{m}^{-1} \cdot \mathrm{s}^{-2}\right]$ & 22.798541 & 52.932635 \\
\hline$u_{\tau}\left[\mathrm{m} \cdot \mathrm{s}^{-1}\right]$ & 4.796121 & 9.187901 \\
\hline$c_{f}[1]$ & 0.001655 & 0.001774 \\
\hline
\end{tabular}

\section{Results analysis}

The transition to turbulence in the boundary layer on the planar plate is a process that is very well described by Morkovin [4] and, of course, by Schlichting [5]. On the emerging, initially stable laminar boundary layer, unstable (Tollmien-Schlichting) waves gradually begin to occur after reaching the $R e_{k r}$, which gradually passes 
into three-dimensional vortices (the so-called lambdastructures). These vortices begin to disintegrate, turbulent areas are formed, and turbulent flow begins to develop.

On a bent plate (airfoil, blade cascades), this mechanism is additionally subjected to a different pressure gradient that can accelerate or delay the process of transition of the boundary layer.

If any irregularity, impurity, etc. occurs on the surface, this may cause a local wedge of a turbulent boundary layer (figure 3), if the impurity is sufficiently large.

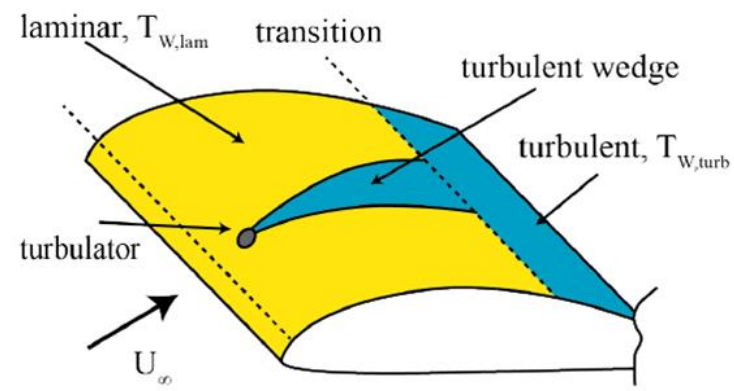

Fig. 3. Scheme of transition of the boundary layer on the airfoil with the representation of the turbulization wedge.

In the images obtained using IR thermography, it is possible to distinguish different color shades that represent the temperature that the surface of the examined model emits at each point. The darkest shades represent the coldest surface area and the lightest shade the warmest surface area. In some cases where surface visualizations using oil solution were also used, it is also possible to identify areas with higher concentrations of solid particles on the surface.

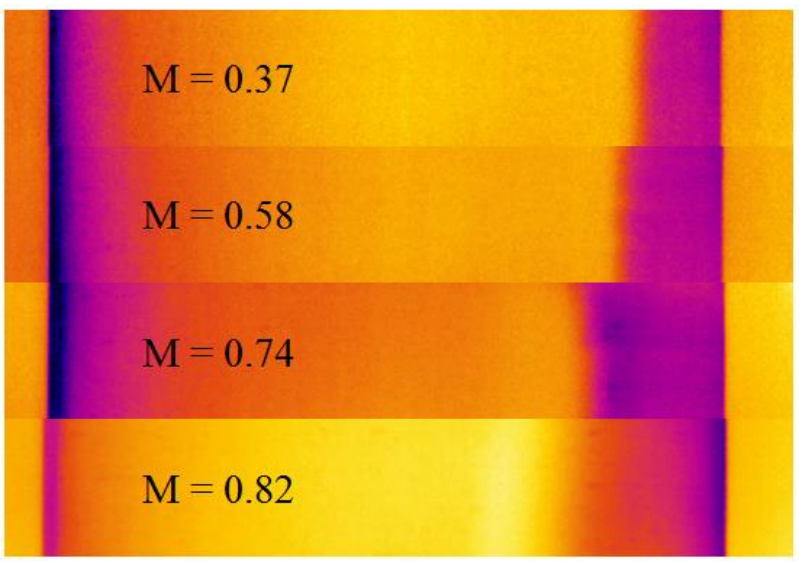

Fig. 4. The comparison of IRT images of the Airfoil without the transition-generator for different Mach numbers.

\subsection{Airfoil without the transition-generator}

Without a transition-generator strip, a total of five regimes were measured for the progressively increasing Mach number: $0.37 ; 0.58 ; 0.74 ; 0.82$ without the use of surface oil visualization. From the results (figure 4), it is obvious that as the Mach number rises, the transition area of the boundary layer moves towards the leading edge.
In the case of transonic regimes, the shock wave oscillates due to the non-stationary tearing of the laminar boundary layer and its position is unclear (figure 5). For the cases of the turbulent boundary layer, the shock wave is considerably more stable (figure 6).

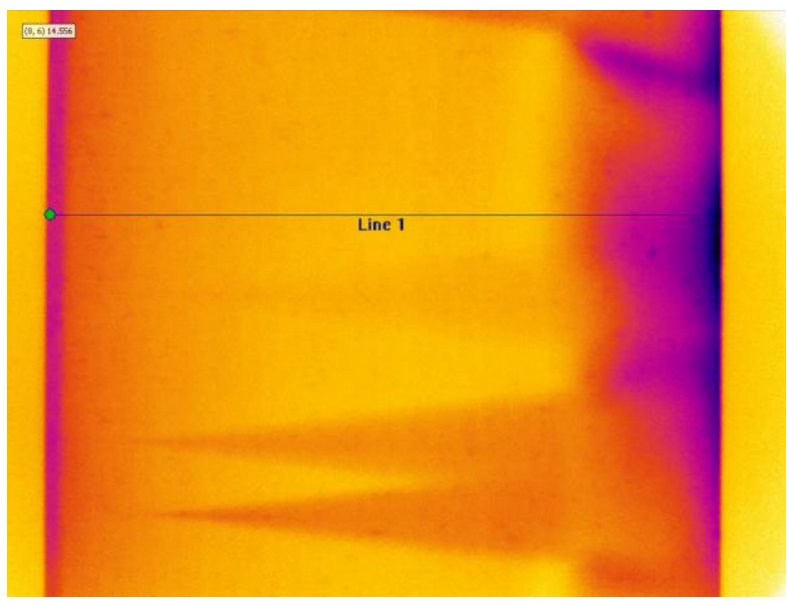

Fig. 5. The IRT im age of the Airfoil without the transition-generator strip $(M=0.8)$

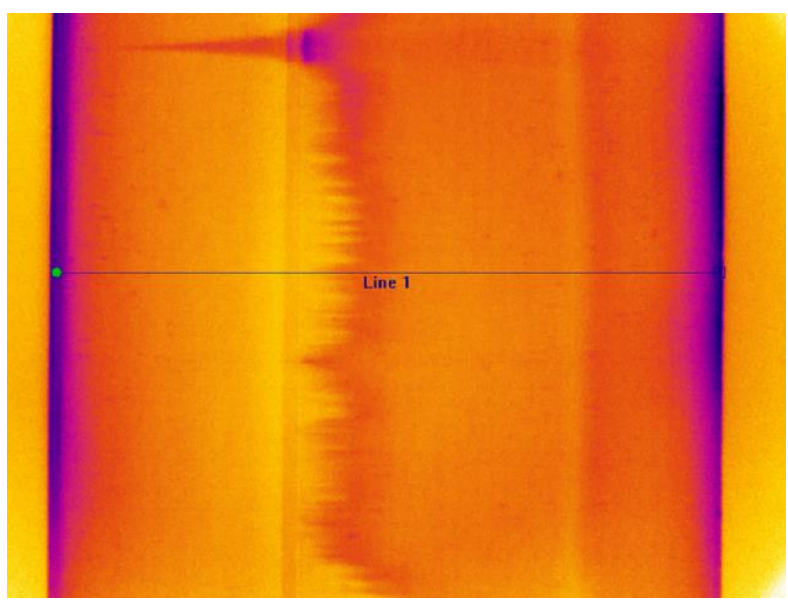

Fig. 6. The IRT im age of the Airfoil with the transitiongenerator strip in $x / c=0,35(M=0.8)$.

\subsection{Comparison of the results for different thicknesses of transition-generator strips}

Figure 7 compares measured data obtained by IR thermography for two Mach number levels and three transition-generator strips thicknesses.

Values of $\mathrm{K}^{\prime}$ and $\mathrm{L}^{\prime}$ are displayed in table 2:

Table 2. Values of $\mathrm{K}^{\prime}$ and $\mathrm{L}^{\prime}$ for tested cases.

\begin{tabular}{llll}
\hline & $M=0.5$ & $M=0.8$ & \\
\hline & $\delta^{*}=0.095$ & $\delta^{*}=0.088$ & $L^{\prime}$ \\
\hline$K=0.04 \mathrm{~mm}$ & $K^{\prime}=0.42$ & $K^{\prime}=0.45$ & 50 \\
$K=0.08 \mathrm{~mm}$ & $K^{\prime}=0.84$ & $K^{\prime}=0.91$ & 25 \\
$K=0.11 \mathrm{~mm}$ & $K^{\prime}=1.16$ & $K^{\prime}=1.25$ & 18 \\
\hline
\end{tabular}

From the results obtained by the IR thermographs, it is evident that even the lowest value of the thickness $\left(K^{\prime}\right.$ $=0.42$ ) causes a transition in the boundary layer. If the 
thickness further increased $\left(K^{\prime}=0.45,0.84,0.91\right)$, the transition area is gradually shortened. The same can be observed when increasing Mach number what means reducing the thickness of the boundary layer under the same conditions, thus increasing the value of $K^{\prime}$.

For values of $K^{\prime}>1$ then we can observe the local boundary layer separation (bubble) behind the transitiongenerator strip. Behind this bubble, there is an almost immediate transition to turbulence (figure 7, e,f).

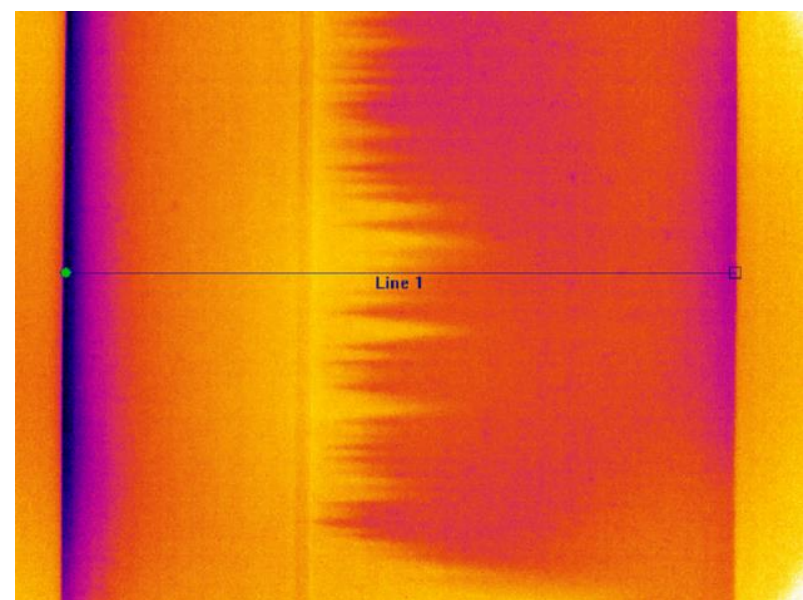

a: $K^{\prime}=0.42(M=0.5, K=0.04)$

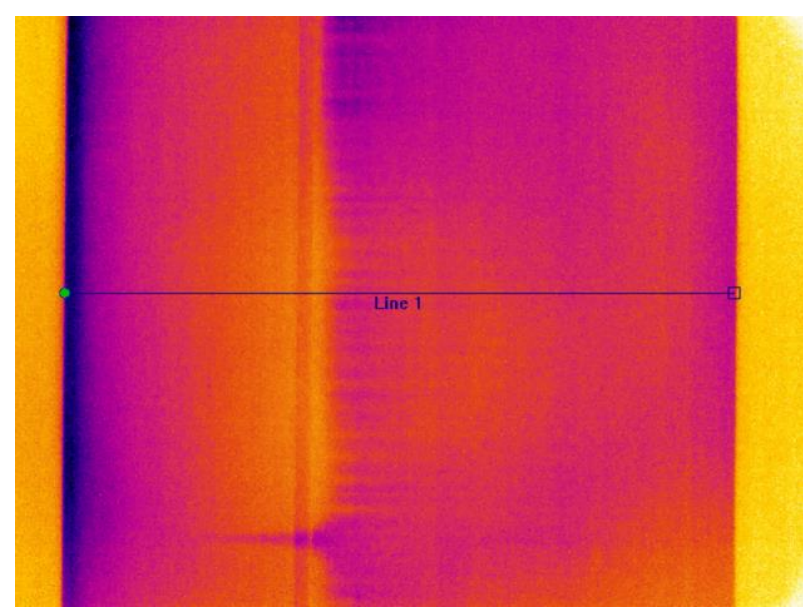

c: $K^{\prime}=0.84(M=0.5, K=0.08)$

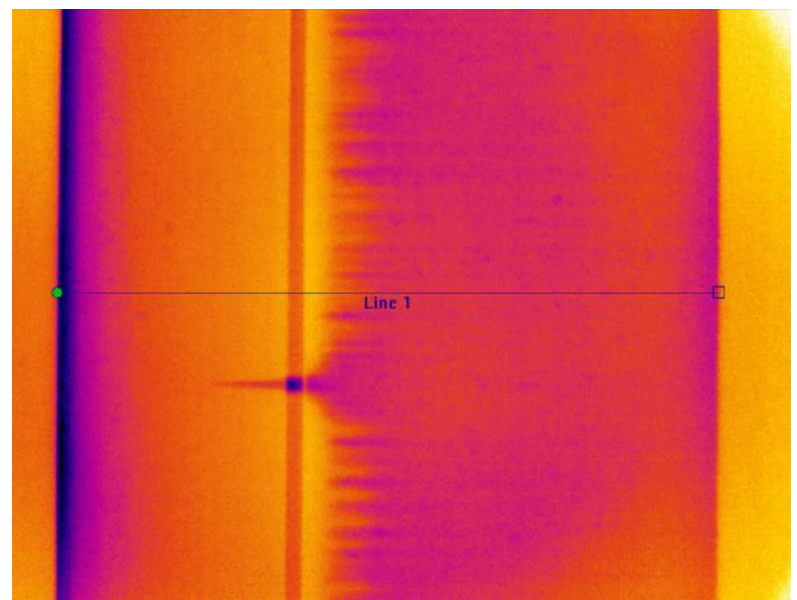

e: $K^{\prime}=1.16(M=0.5, K=0.11)$

Fig. 7 (a, c, e): The IRT im ages of the airfoil with the transition-generator strip in $x / c=0.35$ with various $K$.
In All figures depicted in figure 7, there is a line, indicated as a "Line 1". The temperature profile along the airfoil chord in this line is plotted in figure 8 .

By comparing the temperature profiles along the airfoil chord for the individual investigated cases (figure 8 ), we can observe a gradual increase in temperature from the leading edge to the position of the transitiongenerator strip (in $35 \%$ of the chord).

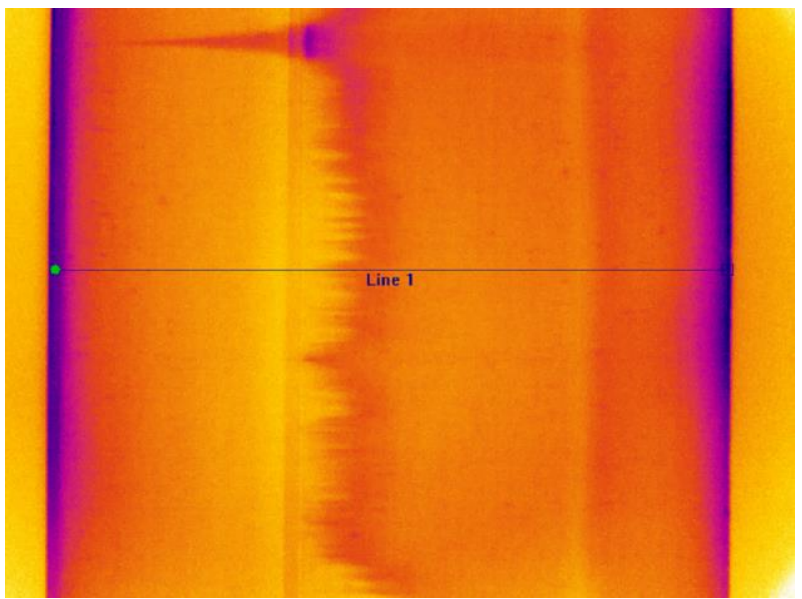

b: $\mathrm{K}^{\prime}=0.45(\mathrm{M}=0.8, \mathrm{~K}=0.04)$

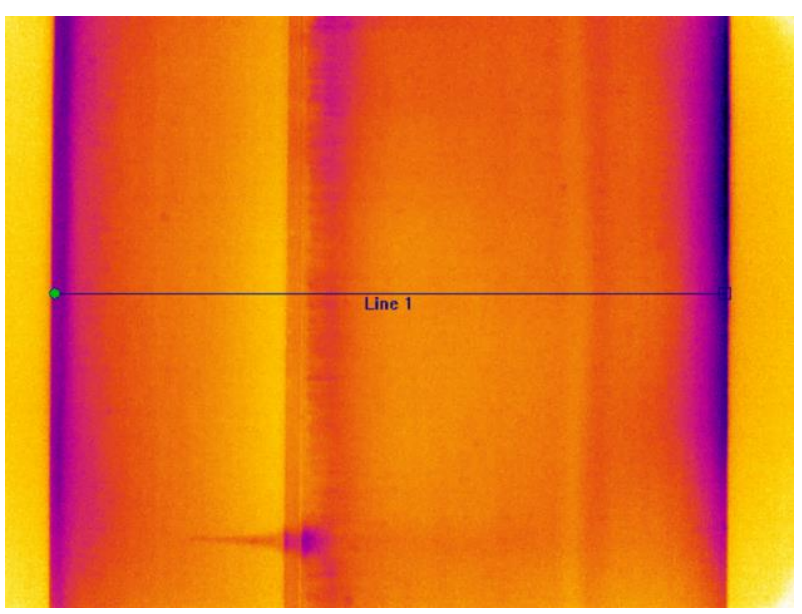

$\mathrm{d}: K^{\prime}=0.91(M=0.8, K=0.08)$

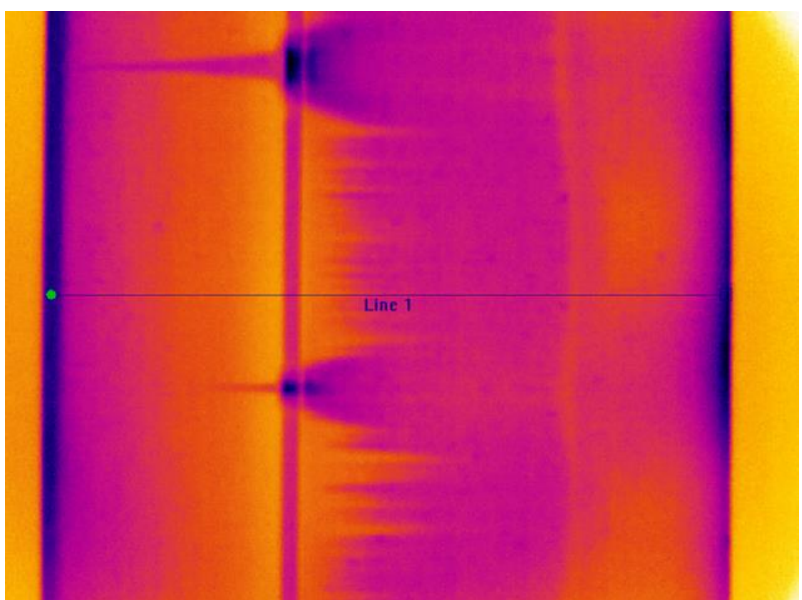

f: $K^{\prime}=1.25(M=0.8, K=0.11)$

Fig. 7 (b, d, f). The IRT im age s of the airfoil with the transition-generator strip in $x / c=0.35$ with various $K$. 
This corresponds to increase the laminar boundary layer thickness, which acts as an insulator and the surface maintains its original temperature.

The subsequent drop in the temperature means the transition area in the boundary layer (turbulization of the boundary layer). Here it can be observed that for the case where $K^{\prime}=0.42$, the transition area is the longest and the most gradual compared to the case where $K^{\prime}=1.25$, when there is a relatively rapid drop in the surface temperature of the model.

A subsequent region of more or less constant temperature means the area of the turbulent boundary layer. If there is a sharp decrease in temperature at the end of the airfoil, it means there is a boundary layer separation.

Schlichting [6] and Tani et al. [7], show the dependence of the surface disparity height $\mathrm{K}$ for the turbulence transition condition (relation of $\operatorname{Re}^{*}{ }_{t}$ over $K / \delta^{*}$ ). This dependency is determined for a single roughness element.

In figure 9 the comparison of the results obtained on the laminar airfoil with the transition-generator strips of different heights with the dependence obtained for the single roughness element is depicted.

It should be noted here that the experiments that led to the determination of dependence on figure 9 and the generalized line according to H.L. Drydena [8], were made assuming the development of a boundary layer on a flat plate, the use of a circular single roughness element and an incompressible flow.

As can be seen in figure 9, the points from the experiment performed in this work are divided by the line of the completed transition in the boundary layer into two areas: For $K^{\prime}=0.42,0.45,0.81$ lies in the unfinished transition area, while values for $K^{\prime}=0.91$, $1.16,1.25$ lies in the transition area.

If we follow the temperature curve in figure 8 , it is evident that for the values of $K^{\prime}=1.25$ and $K^{\prime}=1.16$ there is a clear transition to the boundary layer and the lengths of transition for both cases is roughly $x / c=0.1$. For the case of $K^{\prime}=0.91$, however, the temperature progression indicates a partial relaminarization of the boundary layer after its transition to turbulence.

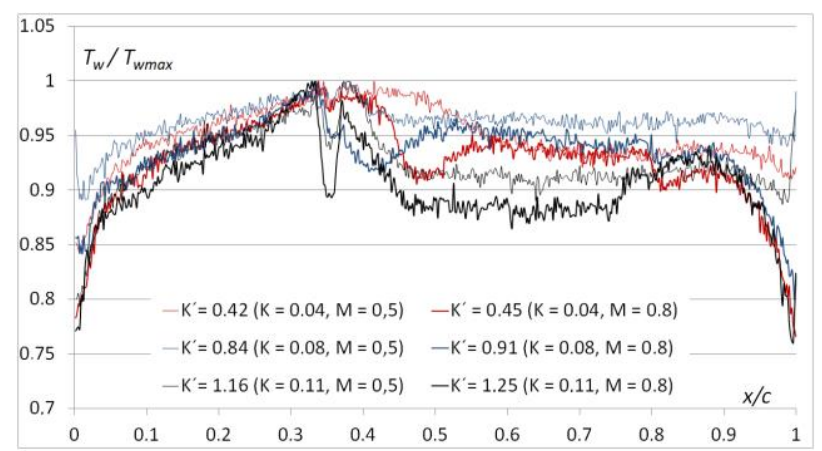

Fig. 8. Temperature profile along the airfoil chord acording to "Line 1" depicted in figure 7.

What can be further observed from the visualization of the transition using IR thermography (in figure 7), is the way of transition itself. It is evident the formation of longitudinal vortices that gradually merge at some distance. In the area of transition, laminar flow lasts in some parts (lighter shades - a warmer surface), in some parts turbulization occurs earlier (darker shades - cooled surface).

This proved the theory of instability in the boundary layer transition. Therefore, if we want to properly predict the transition in the boundary layer using numerical models, it is not appropriate to use simplistic models for 2D numerical calculations since the transition always develops three-dimensionally.

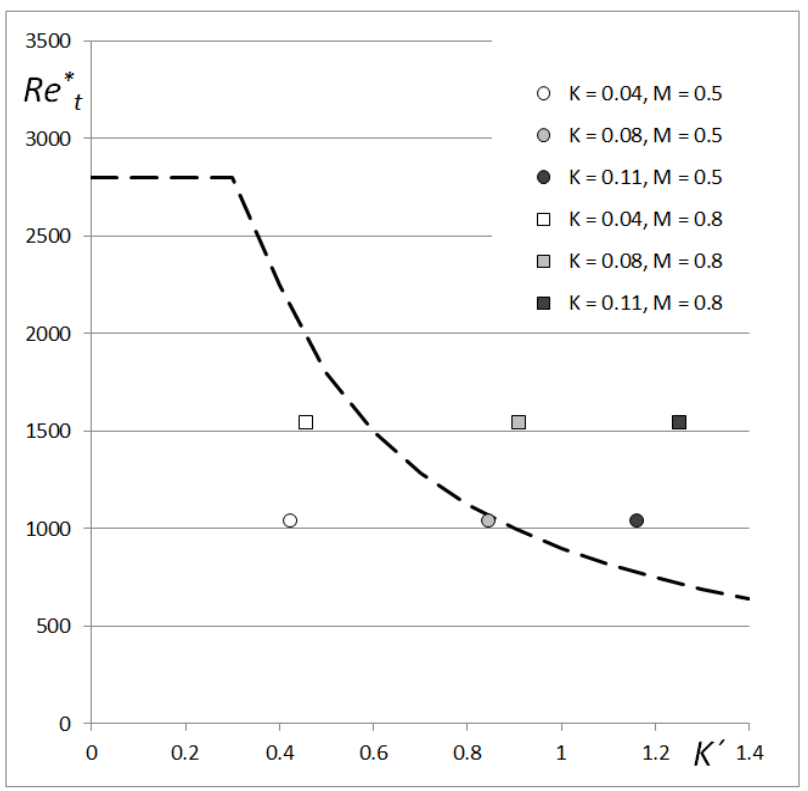

Fig. 9. Effect of roughness element on transition: the dashed line is a transition trashold defined by Tani et al. [7], the points from experiment are inserted.

\section{Conclusions}

The results in this paper demonstrate the violence transition of the boundary layer on a laminar airfoil using the flat roughness element. There is shown how the transition-generator height can influence the boundary layer transition.

The results are compared with well-known dependence of single roughness element and some differences were observed.

The IR thermography was verified for such experiment in high subsonic and transonic Mach numbers application. It nicely visualized the transition region without any surface influence.

The continuation of this work is to use this turbulence generator in a prismatic blade cascade to force the transition of boundary layer in front of the channel throat.

\section{Acknowledgements}

The work was performed with a great acknowledge to TACR for a financial support on the project 
TA04020129 "Investigation of flows in a multi-stage axial turbine stages arrangement".

The work was supported by the Long-term Framework Advancement Plan provided by the Ministry of Industry And Trade of the Czech Republic.

\section{Nomenclature}

c airfoil chord

$c_{f}$ friction coefficient

$h$ heat transfer coefficient

$H$ shape factor of boundary layer

$K \quad$ transition-generator strip thickness

$K^{\prime} \quad$ transition-generator strip thickness related to $\delta^{*}$

$L \quad$ transition-generator strip length

$L^{\prime} \quad$ transition-generator strip length related to $\delta^{*}$

$M \quad$ Mach number

$p$ pressure

$\mathrm{Pr}$ Prandtl number

$q$ heat flow

Re Reynolds number

$R e_{k r}$ Critical Reynolds number:

$R e_{k r}=v_{l} x / v$

Reynolds number at transition point:

$\operatorname{Re}^{*}{ }_{t}=v_{l} \cdot \delta^{*} / v$

$T$ temperature

$u_{\tau} \quad$ friction velocity

$v$ velocity

$x$ coordinate

$y$ coordinate

$\alpha \quad$ angel of attack

$\delta \quad$ boundary layer thickness

$\delta^{*} \quad$ displacement thickness

$\delta^{* *}$ momentum thickness

$\lambda_{c}$ thermal conductivity

$v \quad$ kinematic viscosity

$\tau_{w} \quad$ shear stress

\section{$\underline{\text { Indexes: }}$}

$0 \quad$ total condition

$\infty \quad$ value in far distance in front of the model

max maximum value

$t \quad$ at transition point

$w \quad$ wall condition

\section{References}

1. P. Straka, J. Př́íhoda, Proc. Conf. Experimental Fluid Mechanics, TU Liberec, pp. 636-641, (2010).

2. P. Straka, J. Př́íhoda, P. Śafařík, Proc. Conf. Application of Experimental and Numerical Methods in Fluid Mechanics and Energy, University of Žilina, pp. 261-268, (2012).

3. S. Zuccher, W. S. Saric, Experiments in Fluids, Vol. 44, pp.145-157, (2008)

4. P. Eliasson, Proceedings to Finite Volume for Complex Applications III., ISTE Ltd., London, pp. 527-534, (2002)
5. M. V. Morkovin, Graham R (ed) Transition in turbines. NASA Conf Publ 2386, 161, (1985)

6. Schlichting H., Gersten K., Boundary Layer Theory (8th edd.), Springer-Verlag (2004)

7. I. Tani, at al., Rep. Inst. Sci. Technol. Tokyo University, Vol. 8., (1954)

8. H.L. Drydena, Journal of the Aeronautical Sciences, Vol. 20, 477-482, (1953) 